\title{
Composite fluoropolymer piezoelectric membranes for reconstructive surgery
}

\author{
T T Tverdokhlebova ${ }^{1}$, E N Bolbasov ${ }^{1}$, M Yu Khanova ${ }^{2}$, L V Antonova ${ }^{2}$ \\ and V M Buznik ${ }^{3}$
}

Tomsk Polytechnic University, Tomsk, Russia

Research Institute for Complex Issues of Cardiovascular Diseases, Kemerovo, Russia All Russian Scientific Research Institute of Aviation Materials, Moscow, Russia

E-mail: aramat_tts@mail.ru

\begin{abstract}
The work represents the results of researchers on the formation of composite fluoropolymer piezoelectric membranes based on a copolymer of vinylidene fluoride with tetrafluoroethylene (VDF-TeFE) and polyethylpyrrolidone (PVP) by the electrospinning method. The effect of the PVP content on the structure of the formed membranes was measured using scanning electron microscopy. The biocompatibility of the obtained membranes was studied by fluorescence microscopy on a model of human skin fibroblasts. The studied samples with a 0,5 and $15 \%$ PVP content have good adhesive characteristics, preserve the viability and potential of cells to divide and therefore are most suitable for further use in regenerative medicine.
\end{abstract}

\section{Introduction}

The development of piezoelectric cell membranes ("cell matrixes", scaffolds, matrices) by electrospinning is a promising solution to the problems of reconstructive surgery. The membrane's piezoelectric properties (the ability to induce an electric charge under the influence of external mechanical stresses) allow electrical stimulation of tissue regeneration due to mechanical action on the matrix of surrounding tissues without requiring an external source of electrical energy, implantation of batteries or electrodes, which eliminates the possibility of accumulation of electrolysis products in tissues [1], [2].

VDF-TeFE copolymer is one of the most electroactive polymers with high biological compatibility, chemical resistance, high strength and thermal stability among polymer piezoelectrics. A feature of the VDF-TeFE copolymer is the ability to form polar ferroelectric crystalline phases even during the melt crystallization. The VDF-TeFE ferroelectric properties are due to the dipole moment directed perpendicular to the axis of the polymer chain and arising due to the significantly higher electronegativity of fluorine atoms compared to hydrogen and carbon atoms. One of the main disadvantages limiting the practical use of cell matrices based on the VDF-TeFE copolymer for reconstructive surgery is the high chemical stability and biological inertness due to the chemical structure of this material. The solution to the problems of improving the properties of cell matrices based on the VDF-TeFE copolymer is the development of composite membranes based on thermodynamically immiscible polymers. It is known that the morphology, crystal structure and biocompatibility of composite scaffolds formed by the electrospinning method based on crystallizing polymers are largely 
determined by the ratio of components in the composite scaffold [3]. At present, in the scientific and technical literature there is practically no information on the structure, properties and biocompatibility of promising composite scaffolds based on the VDF-TeFE copolymer depending on the ratio of components in the composite scaffold. Thus, the aim of this research is to study the effect of the ratio of components on the morphology, crystal structure and biocompatibility of composite scaffolds based on the VDF-TeFE copolymer to assess the possibility of their use in the field of reconstructive surgery.

\section{The experimental part}

A VDF-TeFE copolymer (Halopolymer, Russia), polyvinylpyrrolidone (PVP) K-90 (BASF, Germania) and demitylformamide (Ecros-1, Russia) were used to prepare spinning solutions as solvent components. The polymer content in the dope was 5 mass \%. There were produced 5 types of scaffolds for research with $0,5,15,25,50$ mass $\%$ PVP content of the total polymer content in the dope.

Scaffolds were fabricated by electrospinning (NANON 01A MECC Co., Japan). The manufacture's parameters for the scaffolds were described earlier [4].

The morphology and chemical composition of the formed materials was studied using a scanning electron microscope (JEOL JCM-6000, Japan) equipped with an attachment for energy dispersive Xray spectroscopy (JEOL JED 2300, Japan). The average fiber diameter and specific number of defects were calculated using ImageJ 1.5 software (NIH, USA). The crystal structure's studies of the scaffolds were carried out using an XRD 6000 diffractometer (Shimadzu, Japan).

The biocompatibility of the obtained samples was tested by fluorescence microscopy (inverted microscope Axio Observer Z1 (Carl Zeiss, Germany)) on a model of human skin fibroblasts. Fibroblasts were obtained from biopsies of the skin of patients with the method of migration of cells from skin samples placed in Petri dishes. The size of skin biopsies is $1.0 \times 1.0 \mathrm{~cm}$. The fibroblasts obtained during the migration were cultured in DMEM (Sigma-Aldrich) culture medium supplemented with $10 \%$ fetal calf serum (Gibco), 1\% HEPES buffer (Gibco), 1\% L-Glutamine-Penicillin-Streptomycin solution (Gibco) and $0.4 \%$ amphotericin $\mathrm{B}$ (Gibco). Cell cultivation was carried out in a $\mathrm{CO}_{2}$ incubator at $5 \%$ $\mathrm{CO}_{2}, 37^{\circ} \mathrm{C}$ and $95 \%$ humidity.

\section{Results}

The membrane structure's images are presented in Figure 1.

It was found that membranes with a PVP content in the range from 0 to 25 mass $\%$ are formed by interwoven fibers with a normal diameter distribution, have well-developed interconnected porosity (Figure 1), while an increase in PVP content to 25 mass\% leads to a decrease in the average diameter fibers $\left(\mathrm{d}_{\mathrm{av}}\right)$ forming the membrane by more than $20 \%$ (Table 1). The fibers at a PVP content of 50 mass $\%$, become directive, while the tendency to decrease in the average fiber diameter is maintained, which is due to a decrease in the viscosity of the dope with an increase in the PVP content.

Table 1. Average diameter and chemical composition of samples.

\begin{tabular}{cccccc}
\hline PVP & Average & \multicolumn{3}{c}{ Chemical composition [mass \%] } & C/O \\
\cline { 3 - 5 } content [\%] & diameter $[\mu \mathrm{m}]$ & $\mathrm{C}$ & $\mathrm{F}$ & $\mathrm{O}$ & \\
\hline 0 & $0.39 \pm 0.12$ & $47.11 \pm 1.53$ & $51.07 \pm 2.79$ & $1.81 \pm 1.33$ & 26.03 \\
5 & $0.38 \pm 0.10$ & $47.03 \pm 2.66$ & $49.58 \pm 1.44$ & $3.39 \pm 2.43$ & 13.87 \\
15 & $0.37 \pm 0.08$ & $46.80 \pm 1.61$ & $48.67 \pm 0.37$ & $4.52 \pm 1.28$ & 10.35 \\
25 & $0.30 \pm 0.05$ & $51.01 \pm 3.37$ & $40.18 \pm 5.76$ & $8.81 \pm 2.74$ & 5.79 \\
50 & $0.28 \pm 0.06$ & $52.88 \pm 1.25$ & $30.72 \pm 3.76$ & $16.40 \pm 4.97$ & 3.22 \\
\hline
\end{tabular}



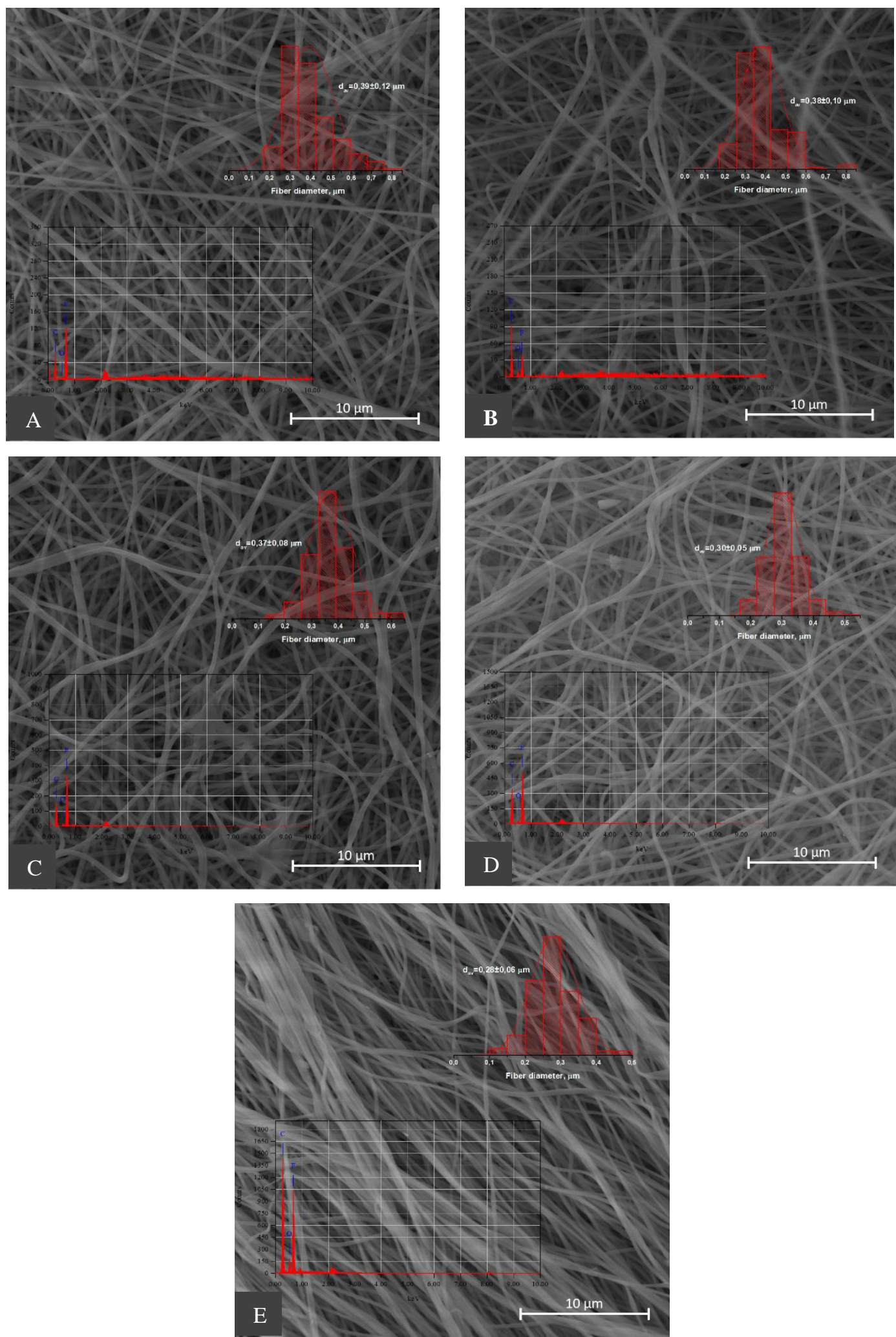

Figure 1. SEM images of samples: A) pure VDF-TeFE; B) VDF-TeFE containing 5\% PVP; C) VDF-TeFE containing 15\% PVP; D) VDF-TeFE containing 25\% PVP; E) VDF-TeFE containing 50\% PVP.

X-ray diffraction patterns of the samples are presented in Figure 2. 


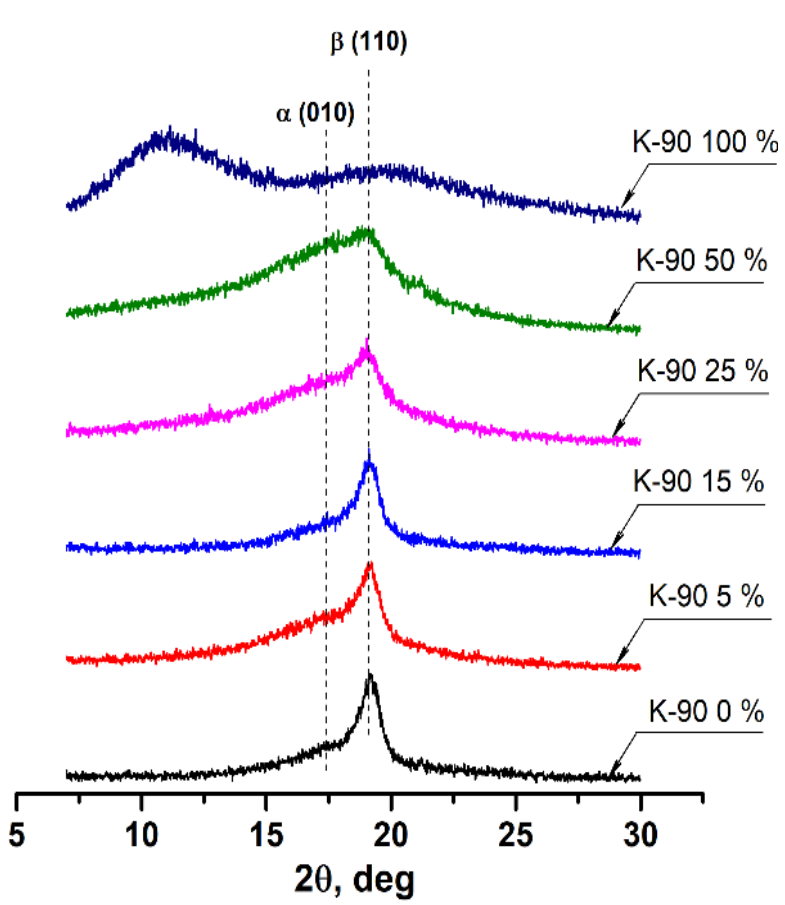

Figure 2. X-ray diffraction patterns of composite scaffolds with different K-90 contents.
In the X-ray diffraction pattern of the scaffold that does not contain K-90 in the region of $19.1^{\circ}$, an intense reflex corresponding to the ferroelectric $\beta$ phase is observed. A slight haloreflex is observed in the region of $17.2^{\circ}$, this is corresponds to the vapor-electric $\alpha$ phase. An increase in the concentration of K-90 in composite scaffolds leads to the intensity of the reflex increase in corresponding to the $\alpha$ phase. In this case the reflex corresponding to the $\beta$ phase is still kept at a high level while the position of the maximum of the reflex does not change (Figure 2). The observed changes indicate increase in the K-90 concentration in composite scaffolds makes it difficult to crystallize the copolymer in the electrically active $\beta$ phase. However, even with a K-90 content of 50 mass $\%$, an electrically active crystalline phase is still formed. Thus the X-ray diffraction analysis results indirectly confirm the presence of piezoelectric properties in composite scaffolds based on the VDF-TeFE copolymer even at high K-90 contents.

There was no significant difference for the absolute number of fibroblasts adhered to the matrix surface $(p>0.05)$ between groups with a PVP content in the range from 0 to $5 \%$. Cells maintained a high level of viability and proliferation. A decrease in the number of adherent and viable fibroblasts was observed at a PVP content of $15 \mathrm{wt} \%$. And the cells in this group had the most pronounced proliferation potential. With an increase in the composition of PVP matrices from 25 to 50 mass $\%$, a fatal cytotoxic effect was obtained in relation to human fibroblasts cultured on matrices. Perhaps this is due to the increased content of dimethylformamide, which forms a toxic complex with K-90.

Table 2. Cytotoxicity of samples.

\begin{tabular}{cccc}
\hline \multirow{2}{*}{$\begin{array}{c}\text { PVP } \\
\text { content [\%] }\end{array}$} & \multicolumn{3}{c}{ In vitro experiment Results $\left(\mathrm{ME}\left(\mathrm{Q}_{1} ; \mathrm{Q}_{3}\right)\right)$} \\
\cline { 2 - 4 } & $\begin{array}{c}\text { The absolute number of } \\
\text { fibroblasts }\left[\mathrm{pcs} / \mathrm{mm}^{2}\right]\end{array}$ & $\begin{array}{c}\text { The absolute number of } \\
\text { viable fibroblasts } \\
{\left[\mathrm{pcs} / \mathrm{mm}^{2}\right]}\end{array}$ & $\begin{array}{c}\text { The relative number of } \\
\text { proliferating cells }[\%]\end{array}$ \\
\hline 0 & $147(92 ; 191)$ & $147(92 ; 191)$ & $16.3(11.2 ; 23.6)$ \\
5 & $141(73 ; 177)$ & $141(73 ; 177)$ & $19.1(14.6 ; 30.8)$ \\
15 & $72(49 ; 92)$ & $69(48 ; 90)$ & $22.5(5.5 ; 41.0)$ \\
25 & $6(3 ; 10)$ & $5(3 ; 9)$ & $11.5(0.0 ; 46.5)$ \\
50 & $8(6 ; 14)$ & $6(4 ; 10)$ & $0.0(0.0 ; 16.3)$ \\
\hline
\end{tabular}

The biocompatibility of polymer matrices with a PVP content in the range from 0 to $25 \%$, it was revealed that the surface of the matrices with a PVP content of 0.5 and $25 \%$ had the highest biocompatibility. Human fibroblasts adhered well to the surface of these matrices maintaining their proliferative potential and viability. After 6 days of cultivation, the absolute number of fibroblasts on the surface of these matrices was similar to the number of cells on the culture plastic. This confirms a similar rate of increase in cell population when cultured on these surfaces. 
Thus, matrices with 0, 5 and 25\% PVP content have good adhesive characteristics, preserve the viability and potential of cells to divide. So they are most suitable for further use in regenerative medicine.
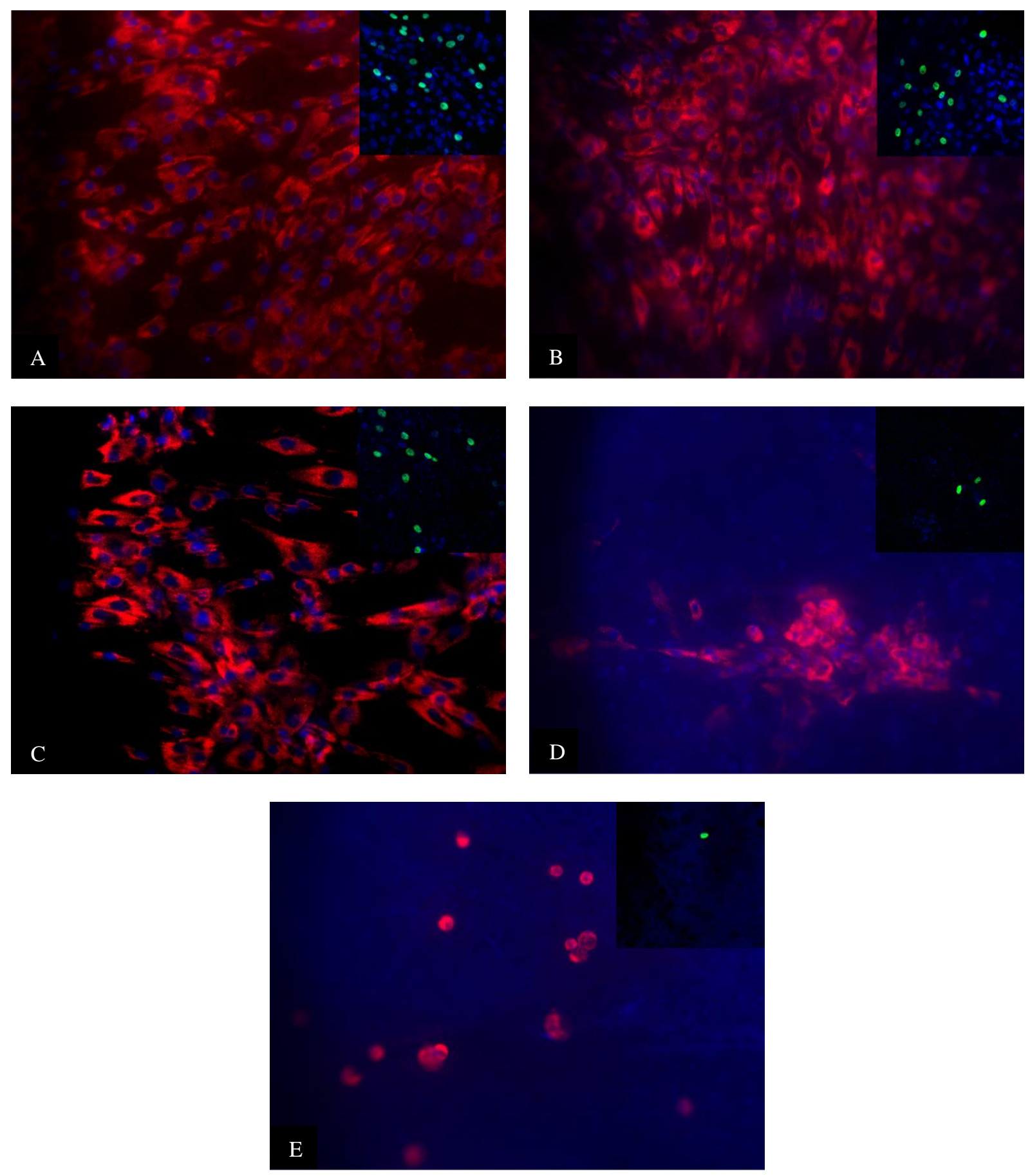

Figure 3. Fluorescence microscopy of human fibroblasts adhered to the surface of prototypes (combination paint PKH26 Hoechst 33342 red-violet, magnification 200) and proliferation of human fibroblasts after 6 days of cultivation on the surface of polymer non-woven membranes (green glow - cell nucleus in the stage of active division, blue glow - nuclei of non-dividing cells, an increase of 200): A) pure VDF-TeFE; B) VDF-TeFE containing 5\% PVP; C) VDF-TeFE containing $15 \%$ PVP; D) VDF-TeFE containing 25\% PVP; E) VDF-TeFE containing 50\% PVP. 


\section{Conclusion}

The possibility of creating composite polymer hydrophilic membranes based on the VDF-TeFE copolymer by electroforming was shown for the first time. It was found that with an increase in the concentration of PVP, the fibers forming the membrane decrease from $0.39 \pm 0.12$ to $0.28 \pm 0.06 \mu \mathrm{m}$. The surface of the matrices with a PVP content in the range from 0 to $15 \%$ was highly biocompatible. Human fibroblasts adhered well to their surface, and in absolute amount, viability and proliferative activity were not inferior to cells cultured on culture plastics. Thus matrices with 0,5 and $15 \% \mathrm{PVP}$ content have high biocompatibility so they are most suitable for further use in regenerative medicine.

Change dimethylformamide with a less toxic solvent is probably a promising method for improving the biocompatibility of the membranes.

\section{Acknowledgments}

The manufacture and study of the physicochemical properties of scaffolds was performed by Tomsk Polytechnic University with the support of RFBR, project number 20-03-00171.

The study of the biocompatibility of scaffolds was supported by the Complex Program of Basic Research under the Siberian Branch of the Russian Academy of Sciences within the Basic Research Topic of Research Institute for Complex Issues of Cardiovascular Diseases № 0546-2019-0002 "Pathogenetic basis for the development of cardiovascular implants from biocompatible materials using patient-oriented approach, mathematical modeling, tissue engineering, and genomic predictors".

\section{References}

[1] B Tandon, J J Blaker and S H 2018 Cartmell Piezoelectric materials as stimulatory biomedical materials and scaffolds for bone repair Acta Biomater. 73 1-20

[2] C Ning, Z Zhou, G Tan, Y Zhu and C Mao 2018 Electroactive polymers for tissue regeneration: Developments and perspectives Prog. Polym. Sci. 81 144-162

[3] Rushikesh S Ambekar, Balasubramanian Kandasubramanian 2019 Advancements in nanofibers for wound dressing: A review European Polymer Journal 117 pp 304-336

[4] T S Tverdokhlebova, E N Bolbasov, V M Bouznik 2020 Composition Polymeric Membranes Based on the VDF-TeFE Copolymer Formed by Electrospinning IOP Conf. Ser. Mater. Sci. Eng. 731012022 